\title{
Efficacy of ICls on patients with oncogene-driven non-small cell lung cancer: a retrospective study
}

\author{
Xiaojin Guo', He Du², Jiayu Li ${ }^{2}$, Menghang Yang ${ }^{2}$, Anweng Xiong ${ }^{2}$, Haiping Zhang ${ }^{2}$, Fengying Wu² \\ 'Department of Immuno-oncology, The Fourth Hospital of Hebei Medical University, Shijiazhuang 050011, Hebei, China. \\ ${ }^{2}$ Department of Medical Oncology, Shanghai Pulmonary Hospital, School of Medicine, Tongji University, Shanghai 200000, \\ China.
}

Correspondence to: Dr. Haiping Zhang, Department of Medical Oncology, Shanghai Pulmonary Hospital, School of Medicine, Tongji University, 507 Zhengmin Road, Shanghai 200000, China. E-mail: zhp7341@sina.com; Dr. Fengying Wu, Department of Medical Oncology, Shanghai Pulmonary Hospital, School of Medicine, Tongji University, 507 Zhengmin Road, Shanghai 200000, China. E-mail: fywu@163.com

How to cite this article: Guo X, Du H, Li J, Yang M, Xiong A, Zhang H, Wu F. Efficacy of ICls on patients with oncogene-driven non-small cell lung cancer: a retrospective study. Cancer Drug Resist 2022;5:15-24. https://dx.doi.org/10.20517/cdr.2021.85

Received: 26 Aug 2021 First Decision: 15 Oct 2021 Revised: 1 Nov 2021 Accepted: 7 Dec 2021 Published: 4 Jan 2022

Academic Editors: Godefridus J. Peters, Chunxia Su Copy Editor: Xi-Jun Chen Production Editor: Xi-Jun Chen

\begin{abstract}
Aim: The objective of our study was to assess the efficacy of immune checkpoint inhibitors (ICls) on patients with non-small-cell lung cancer (NSCLC) harboring oncogenic alterations.

Methods: We retrospectively enrolled patients with advanced non-squamous NSCLC who were treated with antiPD-1-based monotherapy or combined immunotherapy. Major characteristics including PD-L1 expression, treatment, and survival were analyzed.
\end{abstract}

Results: In total, 309 non-squamous NSCLC patients with a median age of 61 years (range 20-88 years) including $70.9 \%$ male were retrospectively enrolled. The molecular alterations involved epidermal growth factor receptor (EGFR) ( $n=81)$, V-Ki-ras2 Kirsten rat sarcoma viral oncogene homolog (KRAS) $(n=31)$, anaplastic lymphoma kinase $(\operatorname{ALK})(n=1)$, human epidermal growth factor receptor 2 (HER2) ( $n=12)$, V-raf murine sarcoma viral oncogene homolog (BRAF) $(n=2)$, rearranged during transfection $(n=4)$, and c-ros oncogene $1(\operatorname{ROS} 1)(n=3)$. In the EGFR subset, the ORR was $30.9 \%(n=81)$ and PFS was significantly shorter than WT group (median PFS: 5.7 months vs. 7.1 months; $P=0.0061$ ). In subgroup analyses, $\mathrm{ICI}$ combined therapy was significantly correlated with a longer PFS compared with ICI monotherapy (median PFS: 7.7 months vs. 4.7 months; $P=0.0112$ ). In KRAS patients, ORR was $51.6 \%(n=31)$. No significant difference was found in subgroup analyses. The ORR and PFS were $16.7 \%$ 
$(n=12)$ and $28.6 \%(n=7), 7.8$ months and 9.0 months for HER2 and EGFR Exon20 insertion patients, respectively. Three ROS1 patients were enrolled with a PFS of 16.0, 34.2, and 45.0 months individually, and one ALK patient with PFS of 4.4 months was identified. No response was found in two BRAF patients.

Conclusion: ICl-based combination therapy can bring benefit to patients with EGFR-mutant NSCLC. ICI-based combination therapy could be considered for patients with ROS1 rearrangement, HER2 mutation and EGFR Exon20 insertion NSCLC.

Keywords: Non-squamous NSCLC, driver mutations, immune checkpoint inhibitor

\section{INTRODUCTION}

Lung cancer is the most common cancer worldwide, in terms of both incidence and mortality ${ }^{[1]}$. Over the past decades, great advancements have been achieved, which are attributed to the understanding of tumor biology and the molecular mechanism of tumor progression. The use of small molecule tyrosine kinase inhibitors (TKIs) has dramatically improved the prognosis of patients with specific genomic aberrations ${ }^{[2-5]}$. However, despite the high response of TKIs, acquired resistance inevitably occurs and limits the long-term benefits ${ }^{[6]}$. Once this happens, the subsequent anti-tumor treatment is limited.

Immune checkpoint inhibitors (ICIs), specifically those targeting PD-1 or programmed death-ligand 1 (PDL1), have rapidly transformed the treatment paradigm for non-small cell lung cancer (NSCLC). For drivernegative NSCLC, ICIs are now the cornerstone of first-line therapy ${ }^{[7]}$. However, whether ICIs alone or in combination with other therapies would bring benefit to those with driver mutations is still to be elucidated. Gainor et al ${ }^{[8]}$ reported poor response of ICI monotherapy in patients with epidermal growth factor receptor (EGFR) mutations or anaplastic lymphoma kinase (ALK) rearrangements. In the phase II ATLANTIC study, durvalumab showed activity in driver-positive NSCLC according to final OS analysis ${ }^{[9]}$. Therefore, continued research is required to explore the optimal use of ICI therapy in patients with driver mutations to improve outcomes of this cohort.

In our study, we retrospectively analyzed patients with locally advanced or metastatic non-squamous NSCLC that were treated with anti-PD-1 based mono- or combined-immunotherapy in Shanghai Pulmonary Hospital to assess the efficacy of ICIs on patients with driver-positive NSCLC.

\section{METHODS}

\section{Study population}

Patients with locally advanced or metastatic non-squamous non-small cell lung cancer from July 2015 to July 2020 in Shanghai Pulmonary Hospital were retrospectively enrolled. Inclusion criteria included the following: (1) a pathologic diagnosis of non-squamous non-small cell lung cancer; (2) testing data (either direct sequencing or NGS on validated platforms) for EGFR, V-Ki-ras2 Kirsten rat sarcoma viral oncogene homolog (KRAS), ALK, human epidermal growth factor receptor 2 (HER2), V-raf murine sarcoma viral oncogene homolog (BRAF), rearranged during transfection (RET), and c-ros oncogene 1 (ROS1); and (3) anti-PD-1-based monotherapy or combined immunotherapy as first-line or posterior-line therapy. Patients treated with fewer than two circles of immunotherapies and had no available complete medical records were excluded. The patient screening process is shown in Figure 1. We reviewed the medical records and abstracted the following patient characteristics: age, gender, Eastern Cooperative Oncology Group Performance Status (ECOG-PS), smoking history, histological type, clinical stage, mutation type, PD-L1 expression, details of treatment, and survival. 


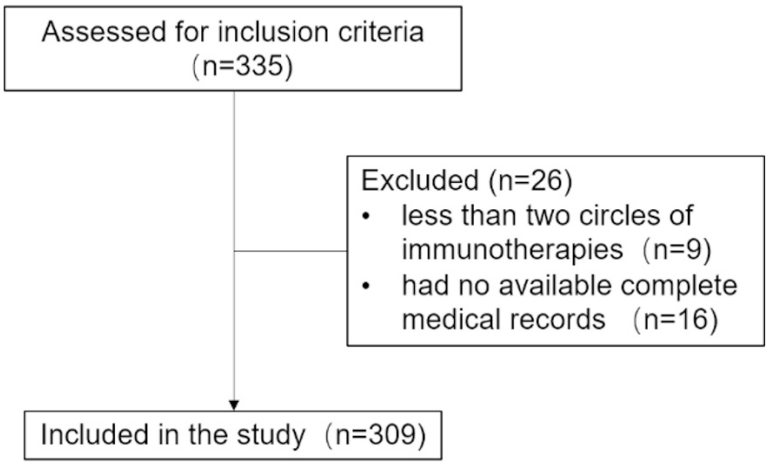

Figure 1. Flow chart of patient screening.

\section{PD-L1 analysis}

PD-L1 immunohistochemistry was performed in Department of Pathology, Shanghai Pulmonary Hospital according to routine procedure. The antibody used was PD-L1 22C3 pharmDx (Dako, Carpinteria, CA, USA). PD-L1 positivity was defined as tumor proportion score cutoff of $1 \%$.

\section{Statistical analysis}

Patient characteristics are expressed as median and range for continuous variables and as frequencies and percentages for categorical variables. Progression-free survival (PFS) was measured from the date of first administration of PD-1 inhibitor treatment to disease progression or death due to any cause or last followup. Response Evaluation Criteria in Solid Tumors guidelines 1.1 (RECIST 1.1) was used to assess tumor response. The difference of response rate among different groups was calculated using Kruskal-Wallis test. Survival data were estimated using the Kaplan-Meier method and compared using the log-rank test in the overall cohort and subgroups. The data were analyzed using GraphPad Prism 8.0 and SPSS Statistics 20. A P value $<0.05$ was considered statistically significant.

\section{RESULTS}

\section{Patient characteristics}

In this study, 309 non-squamous non-small cell lung cancer patients treated with anti-PD-1 based monotherapy or combined immunotherapy in Shanghai Pulmonary Hospital were identified. The median age was 61 years (range 20-88 years), 70.9\% were male (219/309), and 55.7\% (112/309) were never smokers. The majority of the patients had adenocarcinoma histology $(98.4 \%, 304 / 309)$ and ECOG-PS of 0-1 at the start of immunotherapy $(83.5 \%, 258 / 309)$. Detailed characteristics are shown in Table 1. In total, 182 received anti-PD-1 combined with chemotherapy (pemetrexed combined with carboplatin 85.7\%, 156/182; paclitaxel-based chemotherapy $14.3 \%, 26 / 182), 42$ received anti-PD-1 combined with antiangiogenic therapy (apatinib 90.5\%, 38/42; bevacizumab 9.5\%, 4/42). None of the enrolled patients received a combination of ICIs, chemotherapy, and antiangiogenic therapy. Among the whole cohort, 134 had genetic alteration, which involved EGFR $(n=81)$, KRAS $(n=31)$, ALK $(n=1), \operatorname{HER} 2(n=12), \operatorname{BRAF}(n=2), \operatorname{RET}$ $(n=4)$, and ROS1 $(n=3)$. Among the 81 EGFR mutation patients, 42 had exon 19 deletions, 29 had exon20 L858R, and 10 had other mutations; 14 were harboring T790M mutation at the same time and 67 were not. The details of each subgroup are shown in Table 1.

\section{PD-L1 expression}

PD-L1 expression status was available for 132 patients, of whom 54.5\% (72/132) had less than 1\% PD-L1 expression, $31.1 \%(41 / 72)$ had PD-L1 expression of 1\%-49\%, and $14.4 \%(19 / 132)$ had PD-L1 expression more than 50\% [Figure 2A]. Looking into each subgroup, patients with EGFR mutation seemed to have a 
Table 1. Characteristics of patients according to molecular alterations

\begin{tabular}{|c|c|c|c|c|c|c|c|c|c|c|c|}
\hline & \multirow[t]{2}{*}{ All cases } & \multicolumn{3}{|c|}{ EGFR } & \multicolumn{2}{|c|}{ KRAS } & \multirow{2}{*}{$\begin{array}{l}\text { ALK } \\
\text { EML4-ALK }\end{array}$} & \multirow{2}{*}{$\begin{array}{l}\text { HER2 } \\
\text { 20ins }\end{array}$} & \multirow{2}{*}{$\begin{array}{l}\text { BRAF } \\
\text { V600E }\end{array}$} & \multirow{2}{*}{$\begin{array}{l}\text { RET } \\
\text { RET-KIF5B }\end{array}$} & \multirow{2}{*}{$\begin{array}{l}\text { ROS1 } \\
\text { CD74-ROS1 }\end{array}$} \\
\hline & & 19del & 20 L858R & Other & G12C & G12D & & & & & \\
\hline & $n=309$ & $n=42$ & $n=29$ & $n=10$ & $n=30$ & $n=1$ & $n=1$ & $n=12$ & $n=2$ & $n=4$ & $n=3$ \\
\hline Age & $61(20-88)$ & $61(20-72)$ & $60(40-75)$ & $57(38-73)$ & $61(49-75)$ & 71 & 62 & $59(45-68)$ & 49,61 & $49(26-61)$ & $41(41-64)$ \\
\hline \multicolumn{12}{|l|}{ Gender } \\
\hline Male & $219(70.9 \%)$ & $24(57.1 \%)$ & $18(62.1 \%)$ & $7(70 \%)$ & $27(90 \%)$ & $1(100 \%)$ & $1(100 \%)$ & $7(58.3 \%)$ & $2(100 \%)$ & $2(50 \%)$ & $1(33.3 \%)$ \\
\hline Female & $90(29.1 \%)$ & $18(42.9 \%)$ & $11(37.9 \%)$ & $3(30 \%)$ & $3(10 \%)$ & $0(0 \%)$ & $0(0 \%)$ & $5(41.7 \%)$ & $0(0 \%)$ & $2(50 \%)$ & $2(66.7 \%)$ \\
\hline \multicolumn{12}{|l|}{ ECOG performance status } \\
\hline $0-1$ & $258(83.5 \%)$ & $36(85.7 \%)$ & $24(82.8 \%)$ & $7(70 \%)$ & $26(86.7 \%)$ & $1(100 \%)$ & $0(0 \%)$ & $11(91.7 \%)$ & $0(0 \%)$ & $3(75 \%)$ & $3(100 \%)$ \\
\hline$\geq 2$ & $51(16.5 \%)$ & $6(14.3 \%)$ & $5(17.2 \%)$ & $3(30 \%)$ & $4(13.3 \%)$ & $0(0 \%)$ & $1(100 \%)$ & $1(8.3 \%)$ & $2(100 \%)$ & $1(25 \%)$ & $0(0 \%)$ \\
\hline \multicolumn{12}{|l|}{ Smoking history } \\
\hline Current or former & $137(44.3 \%)$ & $27(64.3 \%)$ & $22(75.9 \%)$ & $7(70 \%)$ & $11(36.7 \%)$ & $1(100 \%)$ & $0(0 \%)$ & $4(33.3 \%)$ & $1(50 \%)$ & $0(0 \%)$ & $2(66.7 \%)$ \\
\hline Never & $172(55.7 \%)$ & 15 (35.7\%) & $7(24.1 \%)$ & $3(30 \%)$ & $19(63.3 \%)$ & $0(0 \%)$ & $1(100 \%)$ & $8(66.7 \%)$ & $1(50 \%)$ & $4(100 \%)$ & $1(33.3 \%)$ \\
\hline \multicolumn{12}{|l|}{ Histological type } \\
\hline Adenocarcinoma & $304(98.4 \%)$ & $41(97.6 \%)$ & $29(100 \%)$ & $10(100 \%)$ & $30(100 \%)$ & $1(100 \%)$ & $1(100 \%)$ & $11(91.7 \%)$ & $2(100 \%)$ & $4(100 \%)$ & $3(100 \%)$ \\
\hline NOS & $5(0.6 \%)$ & $1(2.4 \%)$ & $0(0 \%)$ & $0(0 \%)$ & $0(0 \%)$ & $0(0 \%)$ & $0(0 \%)$ & $1(8.3 \%)$ & $0(0 \%)$ & $0(0 \%)$ & $0(0 \%)$ \\
\hline \multicolumn{12}{|l|}{ Clinical Stage } \\
\hline Stage IIIB & $2(0.7 \%)$ & $0(0 \%)$ & $1(3.4 \%)$ & $0(0 \%)$ & $0(0 \%)$ & $0(0 \%)$ & $0(0 \%)$ & $0(0 \%)$ & $0(0 \%)$ & $0(0 \%)$ & $0(0 \%)$ \\
\hline Stage IIIC & $5(1.6 \%)$ & $1(2.4 \%)$ & $1(3.4 \%)$ & $0(0 \%)$ & $0(0 \%)$ & $0(0 \%)$ & $0(0 \%)$ & $0(0 \%)$ & $0(0 \%)$ & $0(0 \%)$ & $0(0 \%)$ \\
\hline Stage IV & $302(97.7 \%)$ & $41(97.6 \%)$ & $27(93.1 \%)$ & $10(100 \%)$ & $30(100 \%)$ & $1(100 \%)$ & $1(100 \%)$ & $12(100 \%)$ & $2(100 \%)$ & $4(100 \%)$ & $3(100 \%)$ \\
\hline \multicolumn{12}{|l|}{ PD-L1 expression } \\
\hline$<1 \%$ & $72(23.3 \%)$ & $11(26.2 \%)$ & $5(17.2 \%)$ & $2(20 \%)$ & $5(16.7 \%)$ & $0(0 \%)$ & $0(0 \%)$ & $5(41.7 \%)$ & $0(0 \%)$ & $3(75 \%)$ & $2(66.7 \%)$ \\
\hline $1 \%-49 \%$ & $41(13.3 \%)$ & $2(4.8 \%)$ & $2(6.9 \%)$ & $0(0 \%)$ & $5(16.7 \%)$ & $0(0 \%)$ & $0(0 \%)$ & $2(16.7 \%)$ & $0(0 \%)$ & $0(0 \%)$ & $1(33.3 \%)$ \\
\hline$\geq 50 \%$ & $19(6.1 \%)$ & $3(7.1 \%)$ & $1(3.4 \%)$ & $0(0 \%)$ & $5(16.7 \%)$ & $0(0 \%)$ & $0(0 \%)$ & $0(0 \%)$ & $0(0 \%)$ & $1(25 \%)$ & $0(0 \%)$ \\
\hline Unknown & $177(57.3 \%)$ & $26(61.9 \%)$ & $21(72.4 \%)$ & $8(80 \%)$ & $15(50 \%)$ & $1(100 \%)$ & $1(100 \%)$ & $5(41.7 \%)$ & $2(100 \%)$ & $0(0 \%)$ & $0(0 \%)$ \\
\hline \multicolumn{12}{|l|}{ Line of $\mathrm{ICls}$} \\
\hline 1 & $106(34.3 \%)$ & $1(2.4 \%)$ & $1(3.4 \%)$ & $4(40 \%)$ & $15(50 \%)$ & $0(0 \%)$ & $0(0 \%)$ & $6(50 \%)$ & $0(0 \%)$ & $2(50 \%)$ & $3(100 \%)$ \\
\hline 2 & $127(41.1 \%)$ & $26(21.9 \%)$ & $17(58.6 \%)$ & $2(20 \%)$ & $12(40 \%)$ & $1(100 \%)$ & $0(0 \%)$ & $3(25 \%)$ & $0(0 \%)$ & $2(50 \%)$ & $0(0 \%)$ \\
\hline$\geq 3$ & $76(24.6 \%)$ & $15(35.7 \%)$ & $11(37.9 \%)$ & $4(40 \%)$ & $3(10 \%)$ & $0(0 \%)$ & $1(100 \%)$ & $3(25 \%)$ & $2(100 \%)$ & $0(0 \%)$ & $0(0 \%)$ \\
\hline \multicolumn{12}{|l|}{ Treatment modality } \\
\hline Anti-PD-1 monotherapy & $85(27.5 \%)$ & $2(4.8 \%)$ & $5(17.2 \%)$ & $1(10 \%)$ & $9(30 \%)$ & $1(100 \%)$ & $1(100 \%)$ & $4(33.3 \%)$ & $2(100 \%)$ & $1(25 \%)$ & $0(0 \%)$ \\
\hline Anti-PD-1 plus chemotherapy & $182(58.9 \%)$ & $39(92.9 \%)$ & $22(75.9 \%)$ & $7(70 \%)$ & $17(56.7 \%)$ & $0(0 \%)$ & $0(0 \%)$ & $7(58.3 \%)$ & $0(0 \%)$ & $3(75 \%)$ & $3(100 \%)$ \\
\hline Anti-PD-1 plus antiangiogenic therapy & $42(13.6 \%)$ & $1(2.4 \%)$ & $2(6.9 \%)$ & $2(20 \%)$ & $4(13.3 \%)$ & $0(0 \%)$ & $0(0 \%)$ & $1(8.3 \%)$ & $0(0 \%)$ & $0(0 \%)$ & $0(0 \%)$ \\
\hline
\end{tabular}

ICIs: Immune checkpoint inhibitors: NOS: not otherwise specified; EGFR: epidermal growth factor receptor; KRAS: V-Ki-ras2 Kirsten rat sarcoma viral oncogene homolog: ALK: anaplastic lymphoma kinase; HER2: human epidermal growth factor receptor 2; BRAF: V-raf murine sarcoma viral oncogene homolog; RET: rearranged during transfection; ROS1: c-ros oncogene 1; ECOG: Eastern Cooperative Oncology Group. 
low PD-L1 expression (26 patients: 69.2\%, 18/26 with PD-L1 < 1\%, 15.4\%, 4/26 with PD-L1 1\%-49\%, and 15.4\%, 4/26 with PD-L1 $\geq 50 \%$ ) [Figure 2B]. In the KRAS subgroup, the percentages of PD-L1 < 1\%, 1\%-49\%, and $\geq 50 \%$ were all $33.3 \%(5 / 15)$ [Figure $2 \mathrm{C}$ ]. Of the seven HER2 cases, $71.4 \%(5 / 7)$ had $<1 \%$ PD-L1 expression, while $28.6 \%$ (2/7) had a PD-L1 expression of $1 \%-49 \%$ [Figure $2 \mathrm{D}$ ].

\section{Response rate}

Based on RECIST 1.1, the ORR of the patients with wild type (WT) was 34.9\% and DOR was 50.3\% ( $n=175$ ), while, in the EGFR subset, the ORR was $30.9 \%$ and DOR was $77.8 \%(n=81)$. For KRAS patients, ORR was $51.6 \%$ and DOR was $83.9 \%(n=31)$. The response rates in both the EGFR group and the KRAS group were statistically different from the WT group $(P=0.029$ and $P=0.004$, respectively $)$ [Figure $3 \mathrm{~A}$ ]. In EGFR patients, the ICI combination therapy subset seemed to have better response rate compared to those who received ICI monotherapy $(P=0.020)$ [Figure $3 \mathrm{~B}]$. However, the difference was not found in KRAS patients [Figure 3C]. In 12 HER2 patients, ORR was $16.7 \%$ and DCR was $91.7 \%$. While ORR was $28.6 \%$ and DCR was $85.7 \%$ in EGFR Exon20 insertion patients [Figure $3 \mathrm{~A}]$.

\section{Progression-free survival}

\section{EGFR}

We investigated the outcomes of ICIs on patients with EGFR mutations. PFS of the EGFR subset was significantly shorter than that of the WT group (median PFS: 5.7 months vs. 7.1 months; $P=0.0061)$ [Figure 4A]. Regarding PD-L1 expression, PFS was not significantly different $(P=0.3721)$ [Figure $4 \mathrm{~B}$ ]. ICI combined therapy was significantly correlated with a longer PFS compared with ICI monotherapy (median PFS: 7.7 months $v s .4 .7$ months; $P=0.0112$ ) [Figure 4C]. PFS was 5.5 months in L858R, 5.9 months in 19del, and 9.0 months in Exon20 insertion and other mutations, but the difference was not statistically significant among the three groups $(P=0.3411)$ [Figure $4 \mathrm{D}$ ]. There was no difference in PFS between patients with or without T790M mutation (median PFS: 5.6 months vs. 5.9 months; $P=0.8381$ ) [Figure $4 \mathrm{E}$ ].

\section{KRAS}

KRAS was not associated with a benefit on PFS compared to those harboring no gene alteration (median PFS: 11.0 months $v s$. 7.1 months; $P=0.5714$ ) [Figure 5A]. In subgroup analyses, PD-L1 positive patients seemed to have longer PFS than PD-L1 negative ones, but the difference was not statistically significant (median PFS: 15.8 months vs. 5.6 months; $P=0.0670$ ) [Figure 5B]. ICI combined therapy had no advantage in PFS compared with ICI monotherapy (median PFS: 12 months vs. 7.25 months; $P=0.5714$ ) [Figure 5C]. 


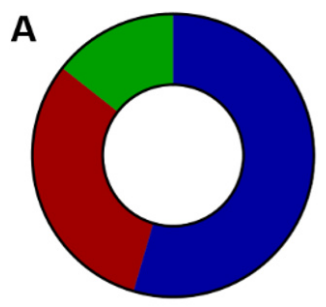

All patients, Total $=132$

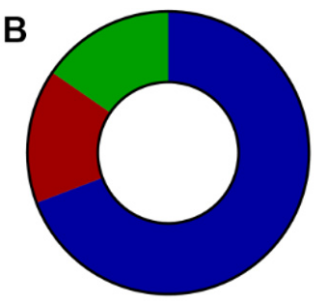

EGFR, Total $=26$

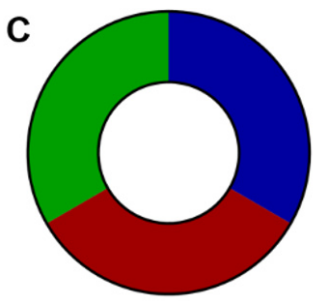

KRAS, Total=15

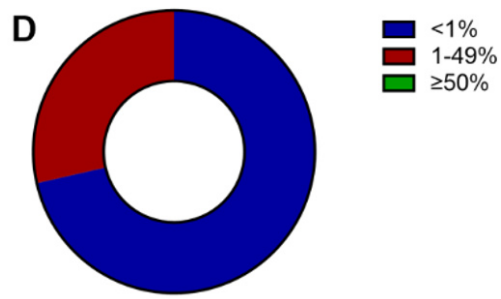

HER2, Total=7

Figure 2. Categorical distribution of tumor PD-L1 expression: the whole cohort (A); EGFR-mutant NSCLC tumors (B); KRAS-mutant NSCLC tumors (C); and HER2-mutant NSCLC tumors (D). EGFR: Epidermal growth factor receptor; NSCLC: non-small-cell lung cancer; KRAS: V-Ki-ras2 Kirsten rat sarcoma viral oncogene homolog; HER2: human epidermal growth factor receptor 2.
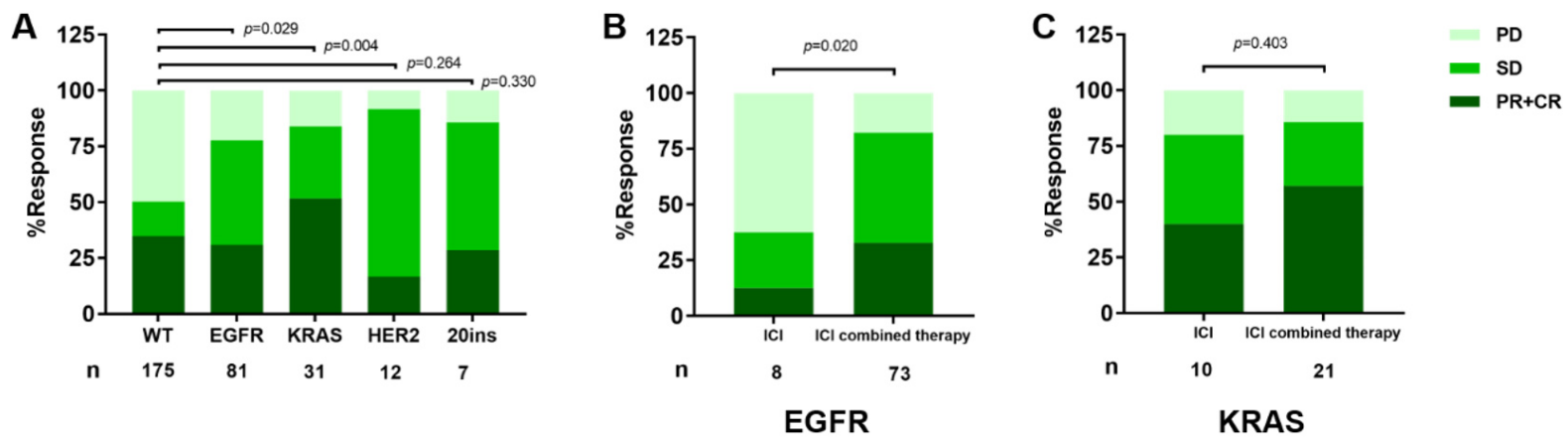

Figure 3. Response to ICls according to Response Evaluation Criteria in Solid Tumors guidelines 1.1 (RECIST 1.1): (A) response to ICls across molecular alterations; (B) response rate by treatment modality in EGFR patients; and (C) response rate by treatment modality in EGFR patients. ICls: Immune checkpoint blockades; PD: progressive disease; SD: stable disease; PR: partial response; CR: complete response; WT: wild type. EGFR: epidermal growth factor receptor; KRAS: V-Ki-ras2 Kirsten rat sarcoma viral oncogene homolog.
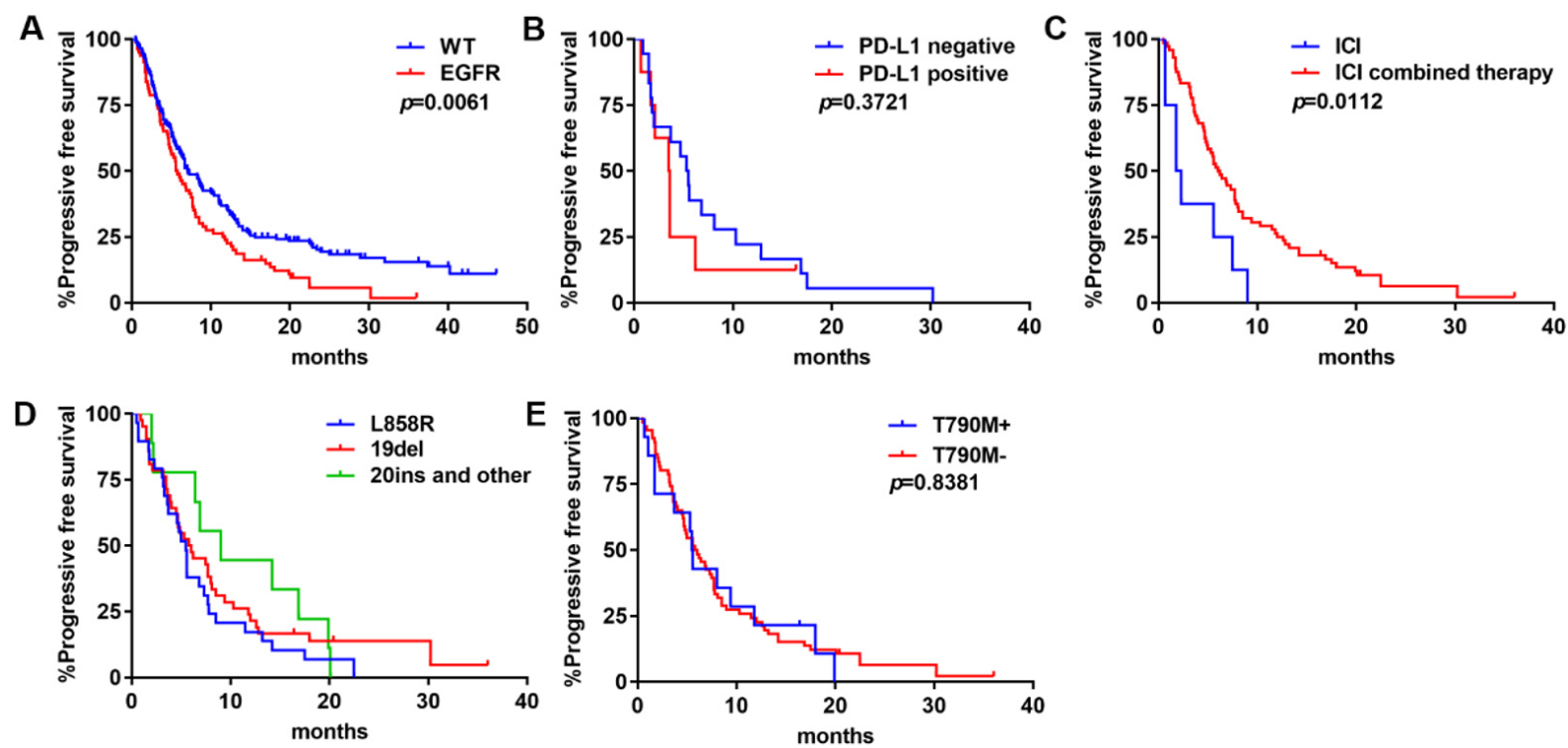

Figure 4. PFS of ICls in EGFR-mutant NSCLC: (A) PFS in tumors with WT NSCLC or EGFR-mutant NSCLC; (B) PFS by PD-L1 expression levels; (C) PFS by treatment modality; (D) PFS by mutation type; and (E) PFS by T790M mutation status. ICls: Immune checkpoint blockades; PFS: progression-free survival; WT: wild type; EGFR: epidermal growth factor receptor; NSCLC: non-small-cell lung cancer. 
A

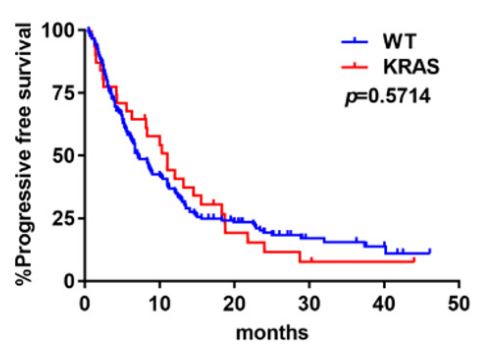

B

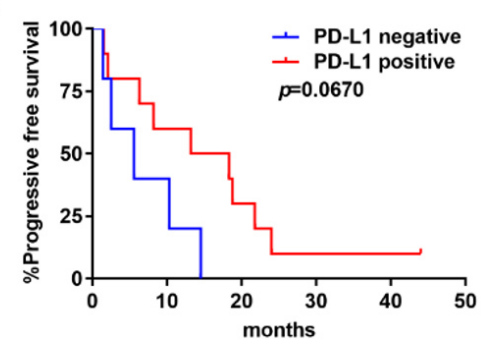

C

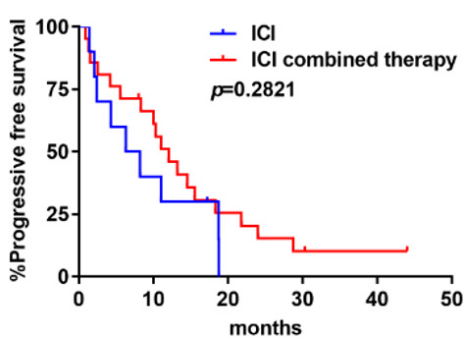

Figure 5. PFS of ICls in KRAS-mutant NSCLC: (A) PFS in tumors with WT NSCLC or KRAS-mutant NSCLC; (B) PFS by PD-L1 expression levels; and (C) PFS by treatment modality. ICls: Immune checkpoint blockades; PFS: progression-free survival; WT: wild type; KRAS: VKi-ras2 Kirsten rat sarcoma viral oncogene homolog; NSCLC: non-small-cell lung cancer.

\section{Other molecular alterations}

All three patients with ROS1 rearrangements and ECOG 1 who received ICIs combined with chemotherapy as first-line treatment had response of PR. The PFS was 16.0, 34.2, and 45.0 months, respectively. The response of the patient with the longest PFS (45 months) was ongoing [Figure 6]. Four patients with RET rearrangements had PFS of 1.5, 1.8, 4.3, and 9.8 months. Three of them had ECOG 1 and one had ECOG 2. They received first-line $(n=2)$ and second-line $(n=2)$ ICI monotherapy $(n=1)$ or ICIs combined with chemotherapy $(n=3)$. No response was found in two BRAF patients, who received after second-line ICI monotherapy. Most HER2 patients had ECOG $1(n=11)$. Seven of them received ICIs combined with chemotherapy, four received ICI monotherapy, and one received ICIs combined with antiangiogenic therapy. ICIs as first-line therapy were observed in six patients, as second-line in three patients, and after second-line in three patients. The median PFS was 7.8 months (range 1.0-26.9 months). ALK rearrangements was identified in only one patient, whose PFS was 4.4 months, with ECOG 3, who received ICI monotherapy as after second-line treatment. We also enrolled seven patients with EGFR Exon20 insertion. The median PFS was nine months (range 2.2-20.1 months). They received ICIs combined with chemotherapy $(n=5)$ or antiangiogenic therapy $(n=1)$ or ICI monotherapy $(n=1)$, as first-line $(n=4)$, second-line $(n=1)$, or after second-line treatment $(n=2)$, with ECOG $1(n=5), 2(n=1)$, or $3(n=1)$.

\section{DISCUSSION}

Targeted therapies against oncogene-driven NSCLC, including $\mathrm{EGFR}^{[2]}, \mathrm{BRAF}^{[10]}$, and HER2 mutations ${ }^{[11]}$ or $\mathrm{ALK}^{[3]}, \mathrm{ROS}_{1}^{[5]}$, and RET ${ }^{[12]}$ rearrangements, have been proved to improve outcomes, and they form the standard first-line treatment in patients with advanced disease. However, progression inevitably occurs, and chemotherapy is currently the main subsequent treatment after TKI resistance. Considering the remarkable long-term benefits of ICI treatments, scientists have made lots of efforts to integrate ICIs into the treatment course of patients with oncogene-driven NSCLC. Mazieres et al. ${ }^{[13]}$ explored the activity of ICIs across NSCLC harboring oncogenic alterations and found driver-positive NSCLC exhibited poor response to ICI monotherapy. Hastings et al. ${ }^{[14]}$ reported more favorable outcomes of exon 21 mutations compared with exon 19 deletions in NSCLC treated with immunotherapy. Until now, the results on this topic are controversial.

In EGFR-driven NSCLC, initial clinical results indicate that ICIs have no clinical benefits. Meta-analysis ${ }^{[15,16]}$ of EGFR-mutated patients in Keynote 010, CheckMate 057, OAK, and POPLAR studies showed ICIs have poorer outcomes in cohorts with EGFR mutations compared to chemotherapy. We found similar findings in our research that patients with EGFR mutations had poor response to ICIs. This may be explained by the immunosuppressive and uninflamed tumor microenvironment (TME) and low tumor mutational burden (TMB), therefore being less immunogenic in the context of oncogenic addiction ${ }^{[17,18]}$. Besides the effect of 

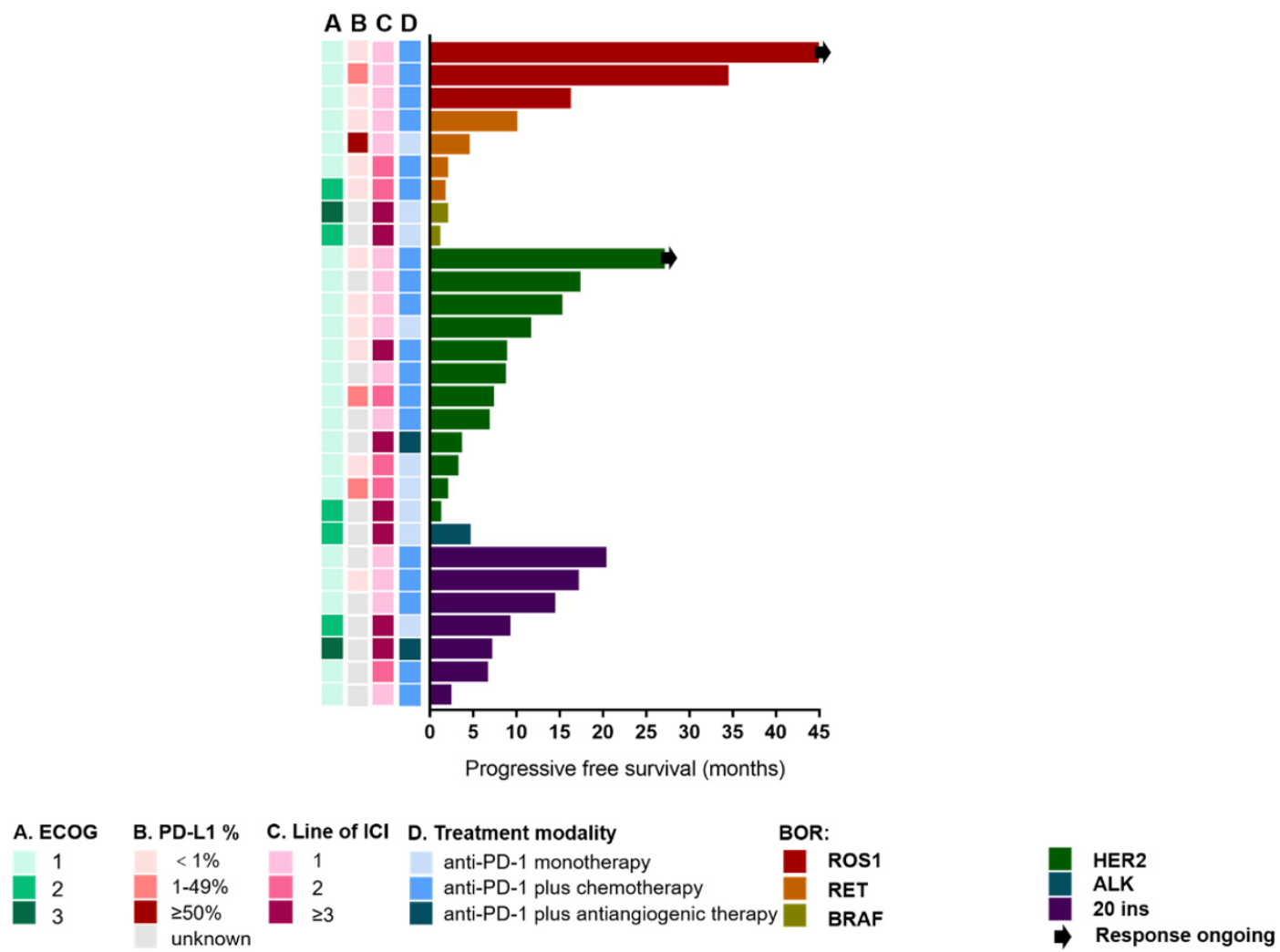

Figure 6. Swimmer's plot of PFS across molecular alterations. PFS: Progression-free survival; ALK: anaplastic lymphoma kinase; HER2: human epidermal growth factor receptor 2; BRAF: V-raf murine sarcoma viral oncogene homolog; RET: rearranged during transfection; ROS1: c-ros oncogene 1; ECOG: Eastern Cooperative Oncology Group; ICl: immune checkpoint blockade.

oncogene mutation on TME, studies have shown that EGFR TKIs, chemotherapy, and antiangiogenic therapy also influence the TME. EGFR TKIs could increase infiltration of CD8+ T cells, dendritic cells, and M1 TAMs and inhibit regulatory $\mathrm{T}$ cells infiltration ${ }^{[19]}$. Bevacizumab, an antiangiogenic therapy, has been observed in melanoma to increase circulating CD8+ T cells and interleukin- 6 levels so as to change the TME in combination with chemotherapy ${ }^{[20]}$. Therefore, the use of ICI-based combined therapy as post-line treatment after TKI resistance may be more effective. In the IMpower150 study, we did see prolonged PFS and OS in the ABCP (atezolizumab, bevacizumab, carboplatin, and paclitaxel) group compared with the BCP (bevacizumab, carboplatin, and paclitaxel) group in patients with EGFR-driven NSCLC ${ }^{[21]}$. In our study, we also found ICI combined therapy was correlated with better outcome than ICI monotherapy in EGFR-driven NSCLC patients.

In our study, we did not find any correlation between KRAS mutation and better survival, which is inconsistent with previous studies. This may be explained by the heterogeneity of KRAS-mutant NSCLC. KRAS mutation subtypes include G12C, G12D, G12V, G12A, and G13D, among others, and different KRAS mutations can activate distinct signaling pathways, leading to different downstream effects, which may result in different response to therapies ${ }^{[22]}$. Besides, KRAS mutation is always accompanied by different patterns of co-occurring mutations, which display different immune profiles and show varying sensitivities to ICIs. In KRAS-TP53 co-mutated tumors, increased expression of PD-L1, higher TMB, and a remarkable clinical benefit of ICIs was observed ${ }^{[2,24]}$. Conversely, KRAS-STK11 has always been associated with poor clinical response to ICIs ${ }^{[2,25]}$. Further studies are needed to differentiate patients suitable for different treatment options. 
HER2 mutations have been reported in approximately $2 \%-5 \%$ of lung adenocarcinomas ${ }^{[26,27]}$ and correlated with poor prognosis ${ }^{[27]}$. The efficacy of ICIs in HER2-mutant NSCLC is ambiguous. Guisier et al. ${ }^{[28]}$ reported 23 patients harboring HER2 mutation treated with ICI monotherapy who had a response rate of $27 \%$, which is close to that observed in unselected patients with NSCLC. Mazieres et al.$^{[13]}$ reported an ORR of $7 \%$ and a median PFS of 2.5 months in 29 patients with HER2 mutation NSCLC treated with ICI monotherapy. In our study, ICI-based therapy displayed certain curative effect with $16.67 \%$ ORR, 91.7\% DCR, and PFS of 7.8 months (range 1-26.9 months). For other rare driver mutations, the efficacy of ICIs on patients with ROS1 rearrangements or EGFR Exon20 insertion was rarely reported. In our study, three patients with ROS1 rearrangements had response of PR and durable PFS of 16.0, 34.2, and 45.0 months. Seven patients with EGFR Exon20 insertion had PFS of 9 months (range 2.2-20.1 months), ORR was 28.6\%, and DCR was $85.7 \%$. Therefore, ICI-based therapy may provide choices for these patients. As to BRAF, RET, and ALK, which represent a small subgroup of NSCLC, because of the limited number of patients, we could not draw a conclusion.

ICI-based combined therapy can bring benefit to patients with EGFR-mutant NSCLC. ICIs, especially ICIbased combination therapy, should not be excluded for patients with ROS1 rearrangement, HER2 mutation, and EGFR Exon20 insertion NSCLC. To achieve maximum benefit for these patients, better predictive biomarkers to select patients and combination modes of therapies should be explored.

\section{DECLARATIONS}

\section{Authors' contributions}

Made substantial contributions to conception and design of the study and performed data analysis and interpretation: Wu F, Zhang $\mathrm{H}$, Guo X

Performed data acquisition, as well as provided administrative, technical, and material support: Du H, Yang M, Li J, Xiong A

\section{Availability of data and materials}

Not applicable.

\section{Financial support and sponsorship}

This work was supported by Clinical Research Plan of SHDC (No. SHDC2020CR4001), and Shanghai Nature Science Foundation (20ZR1447100)

\section{Conflicts of interest}

All authors declared that there are no conflicts of interest.

\section{Ethical approval and consent to participate}

The studies involving human participants were reviewed and approved by The Committee on Medical Ethics of Shanghai Pulmonary Hospital and written informed consent was obtained from all patients.

\section{Consent for publication}

Not applicable.

\section{Copyright}

(c) The Author(s) 2022. 


\section{REFERENCES}

1. Ferlay J, Soerjomataram I, Dikshit R, et al. Cancer incidence and mortality worldwide: sources, methods and major patterns in GLOBOCAN 2012. Int J Cancer 2015;136:E359-86. DOI PubMed

2. Yan K. Osimertinib in EGFR-mutated advanced NSCLC. N Engl J Med 2020;382:1863-5. DOI PubMed

3. Peters S, Camidge DR, Shaw AT, et al; ALEX Trial Investigators. Alectinib versus Crizotinib in untreated ALK-positive non-smallcell lung cancer. N Engl J Med 2017;377:829-38. DOI PubMed

4. Drilon A, Oxnard GR, Tan DSW, et al. Efficacy of selpercatinib in RET fusion-positive non-small-cell lung cancer. $N$ Engl J Med 2020;383:813-24. DOI PubMed PMC

5. Shaw AT, Solomon BJ, Chiari R, et al. Lorlatinib in advanced ROS1-positive non-small-cell lung cancer: a multicentre, open-label, single-arm, phase 1-2 trial. Lancet Oncol 2019;20:1691-701. DOI PubMed

6. Boumahdi S, de Sauvage FJ. The great escape: tumour cell plasticity in resistance to targeted therapy. Nat Rev Drug Discov 2020;19:39-56. DOI PubMed

7. Grant MJ, Herbst RS, Goldberg SB. Selecting the optimal immunotherapy regimen in driver-negative metastatic NSCLC. Nat Rev Clin Oncol 2021;18:625-44. DOI PubMed

8. Gainor JF, Shaw AT, Sequist LV, et al. EGFR mutations and ALK rearrangements are associated with low response rates to PD-1 pathway blockade in non-small cell lung cancer: a retrospective analysis. Clin Cancer Res 2016;22:4585-93. DOI PubMed PMC

9. Garassino MC, Cho BC, Kim JH, et al. Final overall survival and safety update for durvalumab in third- or later-line advanced NSCLC: the phase II ATLANTIC study. Lung Cancer 2020;147:137-42. DOI PubMed

10. Planchard D, Smit EF, Groen HJM, et al. Dabrafenib plus trametinib in patients with previously untreated BRAFV600E-mutant metastatic non-small-cell lung cancer: an open-label, phase 2 trial. Lancet Oncol 2017;18:1307-16. DOI PubMed

11. Koga T, Kobayashi Y, Tomizawa K, et al. Activity of a novel HER2 inhibitor, poziotinib, for HER2 exon 20 mutations in lung cancer and mechanism of acquired resistance: an in vitro study. Lung Cancer 2018;126:72-9. DOI PubMed

12. Ackermann CJ, Stock G, Tay R, Dawod M, Gomes F, Califano R. Targeted therapy for RET-rearranged non-small cell lung cancer: clinical development and future directions. Onco Targets Ther 2019;12:7857-64. DOI PubMed PMC

13. Mazieres J, Drilon A, Lusque A, et al. Immune checkpoint inhibitors for patients with advanced lung cancer and oncogenic driver alterations: results from the IMMUNOTARGET registry. Ann Oncol 2019;30:1321-8. DOI PubMed PMC

14. Hastings $\mathrm{K}$, Yu HA, Wei W, et al. EGFR mutation subtypes and response to immune checkpoint blockade treatment in non-small-cell lung cancer. Ann Oncol 2019;30:1311-20. DOI PubMed PMC

15. Lee CK, Man J, Lord S, et al. Checkpoint inhibitors in metastatic EGFR-mutated non-small cell lung cancer-a meta-analysis. $J$ Thorac Oncol 2017;12:403-7. DOI PubMed

16. Lee CK, Man J, Lord S, et al. Clinical and molecular characteristics associated with survival among patients treated with checkpoint inhibitors for advanced non-small cell lung carcinoma: a systematic review and meta-analysis. JAMA Oncol 2018;4:210-6. DOI PubMed PMC

17. Schrock A, Sharma N, Peled N, et al. MA14.01 updated dataset assessing tumor mutation burden (TMB) as a biomarker for response to PD-1/PD-L1 targeted therapies in lung cancer (LC). J Thorac Oncol 2017;12:S422. DOI

18. Soo RA, Lim SM, Syn NL, et al. Immune checkpoint inhibitors in epidermal growth factor receptor mutant non-small cell lung cancer: current controversies and future directions. Lung Cancer 2018;115:12-20. DOI PubMed

19. Jia Y, Li X, Jiang T, et al. EGFR-targeted therapy alters the tumor microenvironment in EGFR-driven lung tumors: implications for combination therapies. Int J Cancer 2019;145:1432-44. DOI PubMed

20. Mansfield AS, Nevala WK, Lieser EA, Leontovich AA, Markovic SN. The immunomodulatory effects of bevacizumab on systemic immunity in patients with metastatic melanoma. Oncoimmunology 2013;2:e24436. DOI PubMed PMC

21. Reck M, Mok TSK, Nishio M, et al. Atezolizumab plus bevacizumab and chemotherapy in non-small-cell lung cancer (IMpower150): key subgroup analyses of patients with EGFR mutations or baseline liver metastases in a randomised, open-label phase 3 trial. Lancet Resp Med 2019;7:387-401. DOI PubMed

22. Garrido P, Olmedo ME, Gómez A, et al. Treating KRAS-mutant NSCLC: latest evidence and clinical consequences. Ther Adv Med Oncol 2017;9:589-97. DOI PubMed PMC

23. Dong ZY, Zhong WZ, Zhang XC, et al. Potential predictive value of TP53 and KRAS mutation status for response to PD-1 blockade immunotherapy in lung adenocarcinoma. Clin Cancer Res 2017;23:3012-24. DOI PubMed

24. Biton J, Mansuet-Lupo A, Pécuchet N, et al. TP53, STK11, and EGFR mutations predict tumor immune profile and the response to anti-PD-1 in lung adenocarcinoma. Clin Cancer Res 2018;24:5710-23. DOI PubMed

25. Skoulidis F, Goldberg ME, Greenawalt DM, et al. STK11/LKB1 mutations and PD-1 inhibitor resistance in KRAS-mutant lung adenocarcinoma. Cancer Discov 2018;8:822-35. DOI PubMed PMC

26. Genome Atlas Research Network. Comprehensive molecular profiling of lung adenocarcinoma. Nature 2014;511:543-50. DOI

27. Wei XW, Gao X, Zhang XC, et al. Mutational landscape and characteristics of ERBB2 in non-small cell lung cancer. Thorac Cancer 2020;11:1512-21. DOI PubMed PMC

28. Guisier F, Dubos-Arvis C, Viñas F, et al. Efficacy and safety of anti-PD-1 immunotherapy in patients with advanced NSCLC with BRAF, HER2, or MET mutations or RET translocation: GFPC 01-2018. J Thorac Oncol 2020;15:628-36. DOI PubMed 